Ökonomie ist für nachhaltige Entwicklung von zentraler Bedeutung

\title{
Die überfällige Bringschuld der Wirtschaftswissenschaften
}

\author{
Der Klimawandel, die Sicherung der Energieversorgung und \\ die Nachhaltigkeit unseres Konsumverhaltens sind einige der \\ zentralen Herausforderungen unserer Zukunft. Diese Probleme \\ sind nur mit geeigneten ökonomischen Rahmensetzungen zu \\ bewältigen. Ein Interview mit Angelika Zahrnt
}

Der Stern-Report über die Kosten des Klimawandels und der Sukhdev-Bericht über den Wert der Artenvielfalt sind in aller Munde. Welche Rolle spielen derzeit die Wirtschaftswissenschaften bei der Lösung von Nachhaltigkeitsproblemen?

Angelika Zahrnt: Wirtschaftliche Prozesse und die dahinter stehenden Konsummuster sind derzeit die wesentlichen Verursacher von Nachhaltigkeitsproblemen. Der Raubbau als wirtschaftliches Prinzip ist nicht nachhaltig. Auch unter ökonomischen Gesichtspunkten nicht.

Der Stern- und der Sukhdev-Report haben die Aufgabe unternommen, dieses Wissen umfassend zu quantifizieren. Das ist sinnvoll, denn in einer von ökonomischen Argumenten bestimmten Zeit haben quantitative Berechnungen besonderes Gewicht. Dass Ökonomen sich jetzt an die Bezifferung der Folgeschäden des Wirtschaftens machen und aus ökonomischen Gründen für Vorsorgemaßnahmen plädieren, halte ich für eine überfällige Bringschuld dieser Disziplin. Wobei es zuvor schon Ökonomen gab, die hier aktiv waren, aber weder von ihren Kollegen geschätzt noch von Politikern gehört wurden. Das hat sich zum Glück unter dem Problemdruck des Klimawandels geändert.

Welches Potenzial besitzen die Wirtschaftswissenschaften, in der Zukunft eine größere Rolle zu übernehmen?

Angelika Zahrnt: Ich denke, dass in Zukunft neben der Betriebswirtschaft mit ih- rer starken Ausrichtung auf Effizienz die Volkswirtschaft mit ihren gesamtgesellschaftlichen Fragestellungen mehr Gewicht bekommen wird. Allerdings muss gerade auch die Volkswirtschaftslehre ihre bisherige Ausrichtung auf den neoliberalen Mainstream überprüfen. Die ökologische Ökonomie hat jetzt eine große Chance, stärker Einfluss zu nehmen.

Dazu ist es aber notwendig, weitreichende neue Konzepte zu entwickeln: Wie sieht eine steady state economy - eine Wirtschaft, die nicht auf Wirtschaftswachstum ausgerichtet ist - aus, und entsprechende soziale Sicherungssysteme, die nicht vom Wirtschaftswachstum abhängig sind? Wie könnte ein neuer Wirtschaftsindikator aussehen an Stelle des fragwürdigen Bruttoinlandsprodukts? Wie soll die Arbeitswelt neu organisiert werden, wenn Vollbeschäftigung über Wirtschaftswachstum offensichtlich nicht erreicht werden kann? Wie kann Ökonomie nicht nur Markt-Ökonomie, sondern auch die informelle Wirtschaft in den Blick nehmen?

Wie kann ein auf die Freiheitsidee verpflichtetes Gesellschaftssystem eine nachhaltige Entwicklung sicherstellen?

Angelika Zahrnt: Das Ziel von Nachhaltigkeit ist, die ökologische Begrenzung zu respektieren und einen weltweiten Ausgleich zu erreichen mit möglichst vielen Freiheitsgraden für die wirtschaftliche und gesellschaftliche Entwicklung. Aber Grenzen zu respektieren und Klimaziele einzu- halten, kann von manchen Menschen schon als Einschränkung ihrer Freiheit betrachtet werden, zum Beispiel wenn Flugreisen teurer werden. Allerdings - wenn beispielsweise Klimaschutz nicht erfolgreich umgesetzt wird - sind die Freiheiten der Menschen in Küstengebieten, die überschwemmt werden, nicht vorhanden und ihr Leben gefährdet.

Früher bestimmte der Konflikt zwischen Umweltschutz und Beschäftigung die umweltökonomische Debatte. Heute treten zunehmend Konflikte zwischen verschiedenen Umweltschutzzielen, wie zum Beispiel Windenergie und Artenschutz auf.

Welchen Beitrag kann die Ökonomik zur Lösung dieser neuen Konflikte leisten?

Angelika Zahrnt: Zielkonflikte gibt es in Politik, Wirtschaft und persönlichem Alltag. Je komplexer die Probleme sind, desto wichtiger sind transparente Verfahren zur Abwägung. Da können Wirtschaftswissenschaften hilfreich sein. Allerdings können noch so ausgefeilte Kosten-Nutzen-Analysen keine Wertentscheidungen ersetzen. Dies trifft insbesondere auf extreme Szenarien zu, bei denen Unsicherheiten und Kettenreaktionen nicht realistisch einzuschätzen sind. Deshalb muss hier noch stärker das Vorsichtsprinzip zur Geltung kommen, da diese Prozesse nicht umkehrbar sind, wie zum Beispiel beim Klimawandel. Klimaschutz als Vorsorgeprinzip muss in wirtschaftlichen Entscheidungsmechanismen berücksichtigt werden und über ordnungsrechtliche Vorgaben wie zum Beispiel Grenzwerte für den KohlenstoffdioxidAusstoß von Automobilen oder über den Emissionshandel durchgesetzt werden.

Welche Rolle kann der Finanzsektor bei der Bewältigung der Folgen des Klimawandels und im vorbeugenden Klimaschutz spielen?

Angelika Zahrnt: Der Finanzsektor könnte eine wichtige Rolle spielen, indem er in die Bewertung von Finanzanlagen einbezieht, inwieweit eine Firma Teil des Problems oder Teil der Lösung ist. Die Nachfrage nach ethischen Geldanlagen steigt und ist auch eine Chance für den 
Umbau der Wirtschaft in Richtung Nachhaltigkeit.

Die Rückversicherer haben in Deutschland mit ihrer langjährigen statistischen Beobachtung der Schadensfälle eine wichtige Rolle gespielt, als viele den Klimawandel noch für eine Marotte von Umweltschützern hielten. Sie sind jetzt auch engagiert in der Entwicklung neuer Finanz- und Versicherungsprodukte, die Einzelne gegen die Folgen des Klimawandels absichern sollen, gerade in den Ländern des Südens.

Individuelle Einstellungen und Verhaltensweisen der Menschen sind ein wesentlicher Einflussfaktor für nachhaltiges Wirtschaften. Welchen Beitrag können die Wirtschaftswissenschaften zur Förderung eines nachhaltigen Konsums leisten?

Angelika Zahrnt: Auch Ökonomen haben sich inzwischen von der Modellfigur des homo oeconomicus, des rational entscheidenden Verbrauchers, verabschiedet und die Vielfalt von Einflussgrößen beim Kaufentscheid anerkannt. Trotzdem bleibt der Preis ein zentraler Faktor, der allerdings externe Kosten einbeziehen sollte. Zudem sind der ganze Lebenszyklus und Faktoren wie Reparierfähigkeit, Aufrüstmöglichkeiten et cetera zu berücksichtigen.

Der Beitrag der Wirtschaftswissenschaften für eine Verstärkung des nachhaltigen Konsums ist wesentlich in einem Beitrag zu interdisziplinären Projekten mit Soziologen, Psychologen und Praktikern zu sehen. Welche der vielfältigen Einflussfaktoren eine stabile Umorientierung auf nachhaltigen Konsum ermöglichen, ist eine der zentralen Fragen. Ökonomen könnten dazu beitragen, dass der Spruch „Geiz ist geil“ nicht mehr als das Motto eines cleveren smarten Verbrauchers angesehen wird, sondern als Motto eines kurzsichtigen und rücksichtslosen Verbrauchers gilt.

Das Bundesministerium für Bildung und Forschung (BMBF) hat einen Förderschwerpunkt „Wirtschaftswissenschaften für Nachhaltigkeit" ins Leben gerufen. Ökonomen sollen darin durch eine anwendungsbezoge- ne Orientierung praktisch nutzbare Lösungen für Nachhaltigkeitsprobleme liefern.

Welchen Beitrag kann eine solche Förderinitiative zur nachhaltigen Entwicklung in Deutschland leisten?

Angelika Zahrnt: Die Förderinitiative kann das Umsteuern in Richtung Nachhaltigkeit unterstützen, indem das Instrumentarium der Wirtschaftswissenschaften angewandt wird, um neue Zielkonflikte zum Beispiel bei erneuerbaren Energien in ihrer Vielschichtigkeit zu analysieren und um Rückkopplungsprozesse sichtbar zu machen. Auch in Fragen der Optimierung und Risikoabschätzung im Zusammenhang mit nachhaltigen Nutzungsmustern und langfristigen Investitionsentscheidungen können Wirtschaftswissenschaften wichtige Beiträge leisten.

Das bedeutet, Methoden der Wirtschaftswissenschaften auf neue Fragestellungen anzuwenden und auch neue Methoden zu entwickeln. Die Fragestellungen beschäftigen sich mit oft vernachlässigten Themen der Ökonomie, wie Langfristigkeit, Vorsorge und Risiko oder Beständen statt Fließgrößen. Eine Herausforderung ist es, in das systemische Denken der Wirtschaftswissenschaftler auch stärker naturwissenschaftliche und gesellschaftliche Zusammenhänge einzubeziehen.

\section{Anmerkung}

Das ungekürzte Interview sowie weitere Informationen zum BMBF-Förderschwerpunkt Wirtschaftswissenschaften für Nachhaltigkeit finden sie im Internet unter: http://www.wi-n.org.

Das Interview führten Ralph Wilhelm \& Thomas Schulz vom Projektträger im Deutschen Zentrum für Luft- und Raumfahrt e.V.

Die Volkswirtin Dr. Angelika Zahrnt ist Ehrenvorsitzende des Bundes für Umwelt und Naturschutz in Deutschland (BUND) und Mitglied im Rat für Nach-

haltige Entwicklung, der die Bundesregierung in ihrer Nachhaltigkeitspolitik berät.

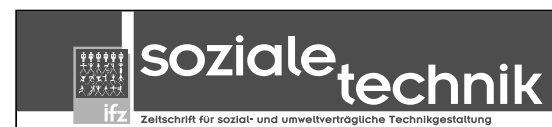

Seit 17 Jahren ist SOZIALE TECHNIK - herausgegeben vom Interuniversitüren Forschungszentrum für Technik, Arbeit und Kultur (IFZ) - ein Forum für die interdisziplinäre Technikforschung und liefert Beiträge für eine sozial- und umweltverträgliche Gestaltung von Technologien. SOZIALE TECHNIK erscheint viertelïhrllich, ein Jahresabonnement kostet $€ 18$; - (für Studierende $€ 13$,-).

Kostenloses Probeabonnement!

\section{Mit Beiträgen zu:}

- Technologie \& Politik

口 Umwelt \& Energie

- Neue Biotechnologien

arauen \& Technik

Informations- \& Kommunikationstechnologien
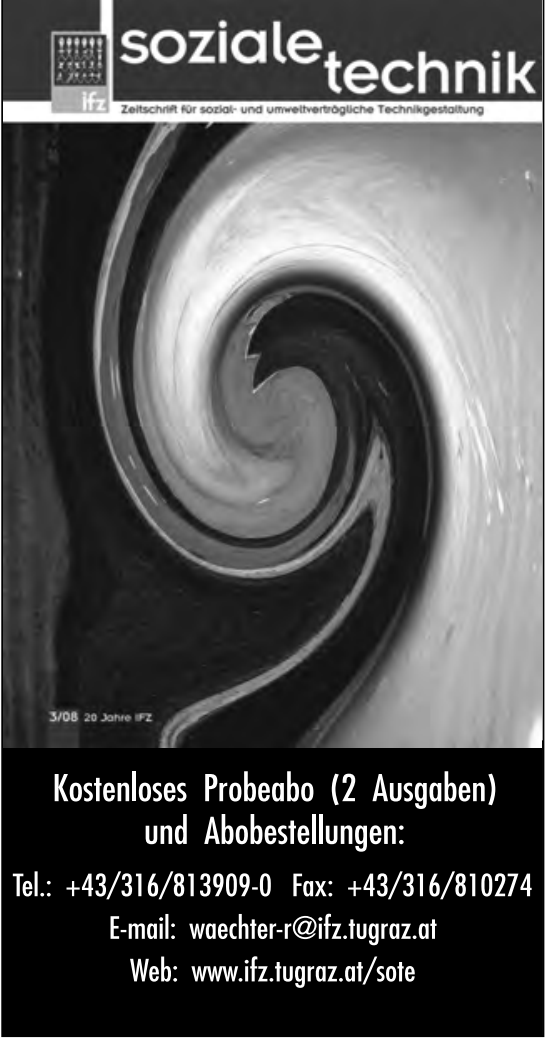
(c) 20I0 Authors; licensee IÖW and oekom verlag. This is an article distributed under the terms of the Creative Commons Attribution Non-Commercial No Derivates License (http://creativecommons.org/licenses/by-nc-nd/3.o/), which permits unrestricted use, distribution, and reproduction in any medium, provided the original work is properly cited. 